\title{
A Study on the Public Awareness of Hierarchical Medical System in Taiwan
}

\author{
Yu-Hua Yan1 (i), Chih-Ming Kung2, Chen-Luan Lu ${ }^{3 *}$ \\ ${ }^{1}$ Superintendent Office, Tainan Municipal Hospital (Managed by Show Chwan Medical Care Corporation), \\ Taiwan \\ ${ }^{2}$ Department of Information Technology and Communication, Shih Chien University Kaohsiung Campus, Taiwan \\ ${ }^{3}$ Medical Affairs Office, Tainan Municipal Hospital (Managed by Show Chwan Medical Care Corporation), Taiwan \\ Email: ^2c0004@mail.tmh.org.tw
}

How to cite this paper: Yan, Y.-H., Kung, C.-M. and Lu, C.-L. (2019) A Study on the Public Awareness of Hierarchical Medical System in Taiwan. Health, 11, 361-370. https://doi.org/10.4236/health.2019.114032

Received: March 3, 2019

Accepted: April 15, 2019

Published: April 18, 2019

Copyright $\odot 2019$ by author(s) and Scientific Research Publishing Inc. This work is licensed under the Creative Commons Attribution International License (CC BY 4.0).

http://creativecommons.org/licenses/by/4.0/

\begin{abstract}
The purpose of hierarchical medical system is to lead in terms of improving efficiency, differentiating healthcare services and promoting labor division by changing the healthcare seeking behavior. The purpose of this research aims to discuss the public awareness of hierarchical medical system in Taiwan for the reference of health policy makers. We obtained our research data using a questionnaire survey; the total number of qualified patients was 1340. This research finds that more subjects agreed to the hierarchical medical system and medical referral system, but many people still disagreed with changes to their healthcare seeking choices due to policy promotion. Subtle changes, therefore, are observed that imply a crisis in terms of the trust in healthcare. The healthcare seeking behavior will not change if there is a difference between the medical awareness of patients and policy implementation, and the government needs to be concerned with this result when making policies.
\end{abstract}

\section{Keywords}

Public Awareness, Hierarchical Medical System, National Health Insurance, Primary Health Care

\section{Introduction}

Over the years, despite National Health Insurance lowering the health care subsidy for the general public, it increased the selectivity when it comes to seeking health care services [1]. There was also a rapid growth in the annual declared medical expenses for outpatient clinics [2]. In general, the growth in the Western outpatient clinical expenses from large hospitals mainly came from increases in 
the number of outpatient clinics. According to study findings, with the exception of those with major illnesses and a need to be referred to larger hospitals for treatment, the majority of the patients could have received their treatment at primary medical institutions.

The partial subsidy provided by the National Health Insurance wasn't adjusted until 2005. Since then, there were no changes for 10 years. In 2017, the National Health Insurance Administration of the Ministry of Health and Welfare $(\mathrm{MOH})$ finally made new arrangements in the co-payment amount, in order to remind the public of the value of medical resources, as well as to promote the health stratification and referral system. The adjustments in the co-payment were solely promoting the hierarchical medical system, changing the publics' behavior going to hospitals for any problem, enhancing the effective use of medical resources and reducing medical resource wastage. The increase in the price of medical care did have an effect on part of the population, making them more willing to go to primary health care facilities first. However, under the current medical health care system in Taiwan, the public still has freedom in making its own choices in terms of choosing health care. It is still an area worth paying attention to for those who are unwilling to cooperate [3].

The key to reform Taiwan's healthcare system is to look for the balanced development of local clinics and hospitals, to implement hierarchical medical system, to establish the family physician system, to reinforce the abilities of whole-person care of physicians and to carry out quality healthcare policies in order to best guarantee the public health [4]. According to the study of Starfield et al., the link of local clinics between the medical transfer system and hierarchical medical system lies in the healthcare responsibility after the initial contact and while the continuous relationship is maintained [5] [6]. Chen [7] found that hierarchical medical system has significant effectiveness in local clinics under the hierarchical medical system. For diseases not treated in local clinics; there have been inconsistencies in healthcare seeking behavior and less significant effectiveness. Compared to those in other cities and counties, residents of Taipei City, with higher monthly premiums, were found to have less significant effectiveness while the number of healthcare seekers had no absolute correlation with the choice of hierarchical medical system. Younger people showed the trend of the decreasing number of healthcare seekers. There was a correlation between the location and frequency of utilization of healthcare resources, indicating the important influence of accessibility on the choice of hierarchical medical system.

Today, Taiwan's National Health Insurance (NHI) program has been proven valuable. It has received high public satisfaction and has been highly praised internationally. Additionally, a Nobel Prize winner even though it was the world's second-best healthcare system and the honor of Taiwan. However, is it sustainable and reasonable that we are able to achieve so much with so little money? Countries from different parts of the world came to learn the lesson from Taiwan and although they gave big thumbs-up to Taiwan's healthcare system, we are puzzled and have not seen any country that completely models our system 
[8]. Politics is always the first priority in Taiwan and it is very difficult to implement hierarchical medical system. Although hierarchical medical system is required by law, according to Article 43 of the National Health Insurance Act (NHIA), the beneficiaries are required to pay 30 percent, 40 percent and 50 percent of the expenses if they visit out-patient departments of district hospitals, regional hospitals and medical centers respectively directly without a referral (self-bearing expenses). Yet, Taiwan's populists are less likely to respect the laws. The simplest goal (implement Article 43 of National Health Insurance Act) is like aiming at the moon and cannot be achieved easily [8]

The purpose of hierarchical medical system is to lead in terms of improving efficiency, differentiating healthcare services and promoting labor division by changing the public's healthcare seeking behavior. Large-scale hospitals, however, believe problems cannot be solved with administrative changes only. Issues such as the equipment at local clinics and the concept change of healthcare seeking behavior shall be addressed and at the same time, healthcare institutions shall work together to establish a more comprehensive system. The Ministry of Health and Welfare proposes to promote hierarchical medical system strategies including the enhancement of services at local clinics, guidance of medical referrals, adjustment of payment standards for critical diseases, reduced services for minor aliments provided by hospitals, enhancement of healthcare cooperation between hospitals and clinics, provision of the continuity of care, and strengthening the management of legal persons [9]. As a result, the purpose of this research aims to discuss the public awareness of hierarchical medical system in Taiwan for the reference of health policy makers, clinics and hospital managers in order to enact a more comprehensive medical referral system.

\section{Methods}

The study subjects were ambulatory patients from hospitals and data was collected between August and December, 2018 from subjects. The basic characteristics of the general public were classified as tendency factors or ability factors. The tendency factors included gender, age, marital status, educational status, occupation and other variables. The ability factors were subdivided into personal or family resources and social resources according to the health behavior model. However, due to the consideration of the national conditions and personal privacy, this study focused on personal and family resources.

After the draft of the scale was completed, three experts in health care administration were invited to conduct expert validation and provide modification suggestions. After the experts reviewed and discussed the questions, eight of the original 20 questions were deleted and 15 questions were left for inspection. The Content Validity Index (CVI) for the appropriateness of the questions was 0.91 .

Oral inquiry was carried out prior to the survey. The survey was done by purposive sampling and the data collection was done by face to face interviews. If the public showed unwillingness or did not fulfill the requirements of the 
questionnaires during any point in time of the survey, under the sampling method, the interviewer could select the next subject. Regarding the representative of the samples, reference is made with the sample size Calculator of Dillman [10]. Calculated with $95 \%$ of the estimated 2300 million as the confidence level, the minimum sample size is 384 .

The total number of qualified patients was 1340. The factors of "gender" and "education" were used to examine the appropriateness in order to prove the effectiveness of the samples and the result showed that these two factors did not have significant influence $(p=0.261 ; p=0.256)$, indicating that each feature is representative. We compiled 10 questions and score by using the Likert 5 point scale from "strongly disagree" (1) to "strongly agree" (5). The higher the score is, the better the hierarchical medical care system and Referral policy. The questionnaire and research design have been inspected and approved by Institutional Review Board by (IRB 1070403) and all subjects have signed the informed consent form.

SPSS for Windows 18.0 was used to create files and analyze research data and the level of significance (p) is $<0.05$. First, the sex, age, marital status, annual income, educational level and occupation. The non-response error verification has been conducted right after questionnaires were received. The questionnaires received were divided into the early respondents and late respondents based by using important constructs (such sex and education level). The result shows that there is no significant difference in these important constructs, and therefore the non-response errors should not affect the sampling of the study.

\section{Results}

\subsection{Personal Characteristics of the General Public}

In terms of the tendency factor, there were more female participants (58.1\%) in this study. The age of the participants ranged from 30 - 39 years old (22.2\%). There were more people with an annual income from 200,001 to 400,000 TWD (26.5\%). In terms of educational status, $59 \%$ of the participants had university qualifications. In terms of occupation, there were more people working for private institutions $(47.0 \%)$, the second being studies (18.8\%). There were more people with an marital status from married (56.3\%). With the Chi-square test, a significant difference has been identified in age, gender, annual income, educational level, and occupation, please see Table 1.

\subsection{Degree of Awareness of the Hierarchical Medical System}

In terms of the awareness of the hierarchical medical system, for the higher payment of healthcare seeking without referrals, 355 (26.5\%) were not aware, $621(46.3 \%)$ partly aware, and $364(27.2 \%)$ very aware of such, respectively, while $538(40.1 \%)$ were not aware, $355(26.5 \%)$ partly aware and 447 (33.4\%) very aware of hierarchical medical system, respectively. For the influence of self-bearing expenses on healthcare seeking choice, 481 (35.9\%) responded no 
and 859 (64.1\%) said yes. For the healthcare institutions that subjects regularly visited, 537 (40.1\%) did not regularly visit specific healthcare providers, while 803 (59.9\%) regularly visited specific healthcare providers. Moreover, among them, 489 (60.9\%) regularly visited local clinics, 109 (13.6\%) district hospitals, $137(17.1 \%)$ regional hospitals and 68 (8.5\%) medical centers, respectively. With the Chi-square test, a significant difference has been identified in for the higher payment of healthcare seeking without referrals, hierarchical medical system, for the influence of self-bearing expenses on healthcare seeking choice and for the healthcare institutions that subjects regularly visited, please see Table 2.

Table 1. Descriptive statistics of personal characteristics $(\mathrm{N}=1340)$.

\begin{tabular}{|c|c|c|c|c|c|c|c|}
\hline Measure & Female & $\%$ & Male & $\%$ & Sum & $\%$ & $\mathrm{X}^{2}$ \\
\hline Age & & & & & & & 0.031 \\
\hline$\leqq 29$ Years & 149 & 11.1 & 76 & 5.7 & 225 & 16.8 & \\
\hline 30 - 39 Years & 180 & 13.4 & 117 & 8.7 & 297 & 22.2 & \\
\hline 40 - 49 Years & 147 & 11.0 & 129 & 9.6 & 276 & 20.6 & \\
\hline $50-59$ Years & 144 & 10.7 & 112 & 8.4 & 256 & 19.1 & \\
\hline$\geqq 60$ Years & 159 & 11.9 & 127 & 9.5 & 286 & 21.3 & \\
\hline Marital status & & & & & & & 0.005 \\
\hline Single & 327 & 24.4 & 191 & 14.3 & 518 & 38.7 & \\
\hline Married & 410 & 30.6 & 345 & 25.7 & 755 & 56.3 & \\
\hline Divorced & 42 & 3.1 & 25 & 1.9 & 67 & 5.0 & \\
\hline Annual income & & & & & & & $<0.001$ \\
\hline No income & 223 & 16.6 & 70 & 5.2 & 293 & 21.9 & \\
\hline$\leqq$ NTD 200,000 & 118 & 8.8 & 62 & 4.6 & 180 & 13.4 & \\
\hline NTD 200,001 - 400,000 & 213 & 15.9 & 142 & 10.6 & 355 & 26.5 & \\
\hline NTD 400,001 - 600,000 & 121 & 9.0 & 155 & 11.6 & 276 & 20.6 & \\
\hline$\geqq$ NTD 600,000 & 104 & 7.8 & 132 & 9.9 & 236 & 17.6 & \\
\hline Educational level & & & & & & & 0.043 \\
\hline Junior high school & 34 & 3.5 & 19 & 1.4 & 53 & 4.0 & \\
\hline Senior high school & 207 & 15.4 & 179 & 13.4 & 386 & 28.8 & \\
\hline University & 481 & 35.9 & 310 & 23.1 & 791 & 59.0 & \\
\hline Graduate School & 57 & 4.3 & 53 & 4.0 & 110 & 8.2 & \\
\hline Occupation & & & & & & & $<.001$ \\
\hline Students & 177 & 13.2 & 75 & 5.6 & 252 & 18.8 & \\
\hline Military and public servants & 55 & 4.1 & 94 & 7.0 & 149 & 11.1 & \\
\hline Private organizations & 340 & 25.4 & 290 & 21.6 & 630 & 47.0 & \\
\hline Worked & 144 & 10.7 & 10 & 0.7 & 154 & 11.5 & \\
\hline Others & 63 & 4.7 & 92 & 6.9 & 155 & 11.6 & \\
\hline
\end{tabular}


Table 2. Gender awareness of the hierarchical medical system.

\begin{tabular}{|c|c|c|c|c|c|c|c|}
\hline Measure & Female & $\%$ & Male & $\%$ & Sum & $\%$ & $\mathrm{X}^{2}$ \\
\hline \multicolumn{7}{|c|}{ For the higher payment of healthcare seeking without referrals } & 0.486 \\
\hline Were not aware & 205 & 15.3 & 150 & 11.2 & 355 & 26.5 & \\
\hline Partly aware & 353 & 26.3 & 268 & 20.0 & 621 & 46.3 & \\
\hline Very aware & 221 & 16.5 & 143 & 10.7 & 364 & 27.2 & \\
\hline \multicolumn{7}{|l|}{ Hierarchical medical system } & 0.018 \\
\hline Were not aware & 312 & 23.3 & 226 & 16.9 & 538 & 40.1 & \\
\hline Partly aware & 187 & 14.0 & 168 & 12.5 & 355 & 26.5 & \\
\hline Very aware & 280 & 20.9 & 167 & 12.5 & 447 & 33.4 & \\
\hline \multicolumn{7}{|c|}{ For the influence of self-bearing expenses on healthcare seeking choice } & 0.065 \\
\hline No & 266 & 19.9 & 215 & 16.0 & 481 & 35.9 & \\
\hline Yes & 513 & 38.3 & 346 & 25.8 & 859 & 64.1 & \\
\hline \multicolumn{7}{|c|}{ For the healthcare institutions that subjects regularly visited } & $<0.001$ \\
\hline No & 265 & 19.8 & 272 & 20.3 & 537 & 40.1 & \\
\hline Yes & 514 & 38.4 & 289 & 21.6 & 803 & 59.9 & \\
\hline Regularly visited & & & & & & & 0.122 \\
\hline Local clinics & 317 & 39.5 & 172 & 21.4 & 489 & 60.9 & \\
\hline District hospitals & 59 & 7.3 & 50 & 6.2 & 109 & 13.6 & \\
\hline Regional hospitals & 93 & 11.6 & 44 & 5.5 & 137 & 17.1 & \\
\hline Medical centers & 45 & 5.6 & 23 & 2.9 & 68 & 8.5 & \\
\hline
\end{tabular}

Second, the current stage of the hierarchical medical system and medical referral system promoted by the government requires patients to seek healthcare at local clinics and 1003 (74.9\%) voiced their agreement and 82 (6.1\%) voiced their disagreement with comments including: I will cooperate with the hierarchical medical system and medical referral system promoted by the government and seek healthcare at local clinics and 1002 (74.7\%) and 108 (8.1\%), respectively; the hierarchical medical system and medical referral system helps improve healthcare quality and 869 (64.8\%) and 124 (9.3\%), respectively; via medical referrals, patients can seek healthcare rapidly and more effectively and $842(64.8 \%)$ and 163 (12.1\%), respectively; medical referrals reduce the waiting time for healthcare and $851(63.5 \%)$ and $179(13.4 \%)$, respectively; healthcare seeking behavior will be changed along with the limited visits to large hospitals and 623 (46.5\%) and 394 (29.4\%), respectively; the NHI policy limits the right to choose healthcare providers and $564(42.5 \%)$ and $368(27.5 \%)$, respectively; without medical referrals, self-bearing expenses will increase and 645 (48.1\%) and 275 (20.5\%), respectively; the hierarchical medical system and medical referral system changes my healthcare choices and 390 (29.1\%) and 641 (47.8\%), respectively; and overall, I do not care about the self-bearing expenses and 571 (42.6\%) and 431 (32.1\%), respectively, please see Table 3. 
Table 3. Descriptive statistics of public awareness of hierarchical medical system.

\begin{tabular}{|c|c|c|c|c|c|c|c|c|c|c|c|c|}
\hline Item & $\begin{array}{l}\text { Strongly } \\
\text { Agree }\end{array}$ & $\%$ & Agree & $\%$ & Neutral & $\%$ & $\begin{array}{l}\text { Disagree } \\
\text { Agree }\end{array}$ & $\%$ & $\begin{array}{l}\text { Strongly } \\
\text { Disagree }\end{array}$ & $\%$ & Mean & Ranking \\
\hline $\begin{array}{l}\text { 1) The hierarchical medical system and } \\
\text { medical referral system promoted by } \\
\text { the government requires patients to } \\
\text { seek healthcare at local clinics }\end{array}$ & 300 & 22.4 & 703 & 52.5 & 255 & 19.0 & 74 & 5.5 & 8 & 0.6 & 3.91 & 1 \\
\hline $\begin{array}{l}\text { 2) I will cooperate with the hierarchical } \\
\text { medical system and medical referral } \\
\text { system promoted by the government } \\
\text { and seek healthcare at local clinics }\end{array}$ & 279 & 20.8 & 723 & 54.0 & 230 & 17.2 & 102 & 7.6 & 6 & 0.4 & 3.87 & 2 \\
\hline $\begin{array}{l}\text { 3) The hierarchical medical system and } \\
\text { medical referral system helps improve } \\
\text { healthcare quality }\end{array}$ & 228 & 17.0 & 641 & 47.8 & 347 & 25.9 & 112 & 8.4 & 12 & 0.9 & 3.72 & 3 \\
\hline $\begin{array}{l}\text { 4) Via medical referrals, patients can } \\
\text { seek healthcare rapidly and more } \\
\text { effectively }\end{array}$ & 201 & 15.0 & 641 & 47.8 & 335 & 25.0 & 144 & 10.7 & 19 & 1.4 & 3.64 & 5 \\
\hline $\begin{array}{l}\text { 5) Medical referrals reduce the waiting } \\
\text { time for healthcare }\end{array}$ & 218 & 16.3 & 633 & 47.2 & 310 & 23.1 & 161 & 12.0 & 18 & 1.3 & 3.65 & 4 \\
\hline $\begin{array}{l}\text { 6) Healthcare seeking behavior will be } \\
\text { changed along with the limited visits to } \\
\text { large hospitals }\end{array}$ & 145 & 10.8 & 478 & 35.7 & 323 & 24.1 & 340 & 25.4 & 54 & 4.0 & 3.24 & 7 \\
\hline $\begin{array}{l}\text { 7) The NHI policy limits the right to } \\
\text { choose healthcare providers }\end{array}$ & 122 & 9.1 & 442 & 33.0 & 408 & 30.4 & 324 & 24.2 & 44 & 3.3 & 3.20 & 8 \\
\hline $\begin{array}{l}\text { 8) Without medical referrals, } \\
\text { self-bearing expenses will increase }\end{array}$ & 137 & 10.2 & 508 & 37.9 & 420 & 31.3 & 240 & 17.9 & 35 & 2.6 & 3.35 & 6 \\
\hline $\begin{array}{l}\text { 9) The hierarchical medical system and } \\
\text { medical referral system changes my } \\
\text { healthcare choices }\end{array}$ & 63 & 4.7 & 327 & 24.4 & 309 & 23.1 & 535 & 39.9 & 106 & 7.9 & 2.78 & 10 \\
\hline $\begin{array}{l}\text { 10) Overall, I do not care about the } \\
\text { self-bearing expenses }\end{array}$ & 110 & 8.2 & 461 & 34.4 & 338 & 25.2 & 359 & 26.8 & 72 & 5.4 & 3.13 & 9 \\
\hline
\end{tabular}

\section{Discussion}

This research studies the public awareness of the hierarchical medical system and medical referral system, and its empirical results via the questionnaire found a significant difference regarding the public awareness of the hierarchical medical system and medical transferal system. At present, freedom of healthcare seeking is respected in Taiwan and at the same time, public interest is taken into consideration. In other words, even though patients have freedom in terms of their healthcare seeking choices, with limited medical resources and without proper management, patients choose to seek healthcare at medical centers and a crowding out effect is seen. Patients with critical diseases that really need to be treated cannot receive treatment in time [11].

This research also finds that more subjects agreed to the hierarchical medical system and medical referral system, but many people still disagreed with changes to their healthcare seeking choices due to policy promotion. Subtle changes, therefore, are observed that imply a crisis in terms of the trust in healthcare. The 
healthcare seeking behavior will not change if there is a difference between the medical awareness of patients and policy implementation, and the government needs to be concerned with this result when making policies.

The literature review proved the influences of patient attributes, physician and clinic attributes, and clinic accessibility on medical transfers [12]. This research also discovers that majority of the subjects agreed to cooperate with the hierarchical medical system and medical referral system promoted by the government, and visited local clinics at first, yet about 30\% disagreed with changes to the healthcare seeking behavior due to limited clinic visits to large hospitals. Among them, about $20 \%$ voiced their disapproval for the increase of self-bearing expenses. This finding is similar with that of Lin and Liu [13], showing that without a mandatory medical referral system, the public in Taiwan fully enjoy the freedom of healthcare seeking and with a low burden on upgrading healthcare seeking, regardless of the disease cause and severity, the public prefers to visit specialist physicians or large-scale hospitals. In addition, in comparison to salary and capitation, fee-for-service (FFS) was associated with $9 \%-12 \%$ lower referral rate presumably because physicians wanted to treat patients and increase their incomes by producing more services [14].

\section{Conclusions}

Taiwan's NHI program is a single-payer system and it implements the global budget system. Hence, the negotiation results of the global budget will definitely affect the healthcare ecosystem. There is a need to seriously consider how to get rid of the frame of the global budget to avoid the insufficiency of healthcare resources and resistance to policy implementation.

The hierarchical medical system and medical referral system in Taiwan are not effectively implemented; for patients, even after undergoing surgery, they basically are cared for within several weeks. Only through an effective mechanism and system will the NHI program be sustainable. Taiwan implements the total global budget system, and local clinics and hospitals have their own global budget. All healthcare providers share the same pie of the same global budget and future studies need to discuss whether or not the healthcare seeking behavior changes due to the crowding out effect.

\section{Limitations}

This study has certain limitations. The participants were being randomly chosen and the study was of an anonymous nature, there is no way to ensure that the all respondents follow-up survey. Therefore, the results should be generalized with caution.

\section{Conflicts of Interest}

The authors declare no conflicts of interest regarding the publication of this paper. 


\section{References}

[1] Wen, Y.P., Huang, S.M. and Chiang, T.L. (2012) An Analysis of the Growth of Healthcare Expenditure in Taiwan: Healthcare Inflation, Volume-Intensity, and Equity. Taiwan Journal of Public Health, 31, 1-10.

[2] Lin, J.Y., Yeh, M.K. and Lin, C.R. (2001) Impact of a New Medical Center on Local Area Medical Behavior: Case Study of Nei-Hu. The Journal of Health Science, 3, 330-349.

[3] Tsai, W.C. and Kung, P.T. (2003) Consumers' Assessment of Clinic Services and Its Affecting Factors. Taiwan Journal of Public Health, 22, 181-193.

[4] Chiu, T.Y. (2016) Implementing the Cooperative Mechanism of the Hierarchy of Medical Care-Establishment of the Balanced Medical Care System between Hospitals and Community Medical Units. Taiwan Medical Journal, 59, 405-406. (In Chinese)

[5] Starfield, B. (1994) Is Primary Care Essential? Lancet, 344, 1129-1133. https://doi.org/10.1016/S0140-6736(94)90634-3

[6] Starfield, B. and Fryer, G.E. (2007) The Primary Care Physician Workforce: Ethical and Policy Implications. Annals of Family Medicine, 5, 486-491.

https://doi.org/10.1370/afm.720

[7] Chen, C.C. (2008) A Study of Patients' Medical Seeking Behavior by Data Mining. Unpublished Master's Thesis, Southern Taiwan University of Science and Technology, Taiwan. (In Chinese)

[8] Wang, Y.H. (2017) Grading Medical Treatment and Prescription for the Right Medicine. Taiwan Medical Journal, 60, 38. (In Chinese)

[9] Chang, R.Z. (2017) Medical Safety and Quality Seminar Series: Grading Medical. Taiwan Medical Journal, 60, 22-27. (In Chinese)

[10] Dillman, D. (2000) Constructing the Questionnaire, Mail and Internet Surveys. John Wiley \& Sons, New York.

[11] Chang, T. (2015) A Research of Referral in Taiwan: From Legal Policy, Medical Ethics, and Social Core Value Perspectives. Journal of Yuanpei University of Science and Technology, 22, 1-14. https://lib.ypu.edu.tw/var/file/21/1021/img/1402/635516332.pdf

[12] O’Donnell, C.A. (2000) Variation in GP Referral Rates: What Can We Learn from the Literature? Family Practice, 17, 462-471.

https://doi.org/10.1093/fampra/17.6.462

[13] Lin, P.J. and Liu,Y.C. (2010) Factors Influencing the Return-Visit Rate for Referred Patients in a Community to Their Primary Care Clinics. Taiwan Journal of Public Health, 29, 228-237.

[14] Vahidi, R.G., et al. (2013) A Systematic Review of the Effect of Payment Mechanisms on Family Physicians Service Provision and Referral Rate Behavior. Journal of Pakistan Medical Students, 3, 54-60.

https://www.researchgate.net/publication/280708333_A_Systematic_Review_of_the _Effect_of_Payment_Mechanisms_on_Family_Physicians_Service_Provision_and Referral_Rate_Behavior 


\section{Appendix1: Questionnaires}

[1] For the higher payment of healthcare seeking without referrals?

[2] For the influence of self-bearing expenses on healthcare seeking choice?

[3] For the healthcare institutions that subjects regularly visited?

[4] Is it clear that the hierarchical medical system?

[5] Regularly visited? (Local clinics, District hospitals, Regional hospitals, Medical centers)

[6] The hierarchical medical system and medical referral system promoted by the government requires patients to seek healthcare at local clinics?

[7] I will cooperate with the hierarchical medical system and medical referral system promoted by the government and seek healthcare at local clinics?

[8] The hierarchical medical system and medical referral system helps improve healthcare quality?

[9] Via medical referrals, patients can seek healthcare rapidly and more effectively?

[10] Medical referrals reduce the waiting time for healthcare?

[11] Healthcare seeking behavior will be changed along with the limited visits to large hospitals?

[12] The NHI policy limits the right to choose healthcare providers?

[13] Without medical referrals, self-bearing expenses will increase?

[14] The hierarchical medical system and medical referral system changes my healthcare choices?

[15] Overall, I do not care about the self-bearing expenses? 